\title{
Leitthema
}

Bundesgesundheitsbl 2016 · 59:4-16 DOI 10.1007/s00103-015-2267-6

Online publiziert: 2. November 2015

c) Springer-Verlag Berlin Heidelberg 2015
Cornelia Lange · Anne Starker · Elena von der Lippe · Heike Hölling Abteilung für Epidemiologie nichtübertragbarer Krankheiten und Gesundheitsmonitoring, Robert Koch-Institut, Berlin, Deutschland

\section{Psychische und körperliche Gewalterfahrungen in den vergangenen 12 Monaten in der Allgemeinbevölkerung}

\section{Ergebnisse der Studie zur Gesundheit Erwachsener in Deutschland (DEGS1)}

\section{Einleitung}

Gewalt ist ein vielschichtiges und nicht eindeutig definiertes Phänomen, dessen Bedeutung im öffentlichen Bewusstsein von körperlichen und seelischen Verletzungen über bestimmte Formen von Kriminalität, rüdem Verhalten im Straßenverkehr und Sport bis hin zu sozialer oder politischer Benachteiligung reicht [1]. Zudem unterliegt der Begriff auch einem historischen und sozialen Bedeutungswandel. Aus Public-health-Sicht ist es erforderlich, Gewalt so zu definieren, dass die gesamte Bandbreite der Täterhandlungen und die subjektive Erfahrung der Opfer eingeschlossen sind, ohne aber diese zu bagatellisieren oder Wechselfälle des täglichen Lebens als krankhaft einzustufen [2]. Die Weltgesundheitsorganisation (WHO) definiert Gewalt als „absichtliche[n] Gebrauch von angedrohtem oder tatsächlichem körperlichem Zwang oder physischer Macht gegen die eigene oder eine andere Person, gegen eine Gruppe oder Gemeinschaft, der entweder konkret oder mit hoher Wahrscheinlichkeit zu Verletzungen, Tod, psychischen Schäden, Fehlentwicklung oder Deprivation führt" [3].

Um die Komplexität und Unterschiedlichkeit gewalttätigen Handelns $\mathrm{zu}$ verdeutlichen und einen analytischen Bezugsrahmen zu schaffen, hat die WHO eine Typologie der Gewalt entwickelt.
Dabei wird Gewalt in 3 Kategorien gegliedert, die darauf Bezug nehmen, von wem die Gewalt ausgeht: Unterschieden wird zwischen der Gewalt, die sich eine Person selbst antut, zwischenmenschlicher Gewalt (Gewalt im engeren und weiteren sozialen Nahraum) und kollektiver Gewalt, die von größeren Gruppierungen wie Staaten, organisierten politischen Gruppen oder Terrororganisationen ausgeübt wird. Zudem wird die jeweilige Form der Gewaltausübung konkretisiert (körperliche, psychische, sexuelle Gewalt, Deprivation/Vernachlässigung; • Abb. 1). Mit dieser Typologie werden sowohl die Bedeutung des sozialen Umfelds, die Beziehung zwischen Täter und Opfer und im Fall von kollektiver Gewalt - die möglichen Motive von Gewalt mit berücksichtigt.

Es existieren daneben weitere Kriterien zur differenzierten Beschreibung von Gewaltausübung, z. B. nach Intention $[4,5]$, nach Bedeutung im Geschlechterverhältnis oder Stärke [4-7].

Gewalterfahrungen können bei den Opfern mit erheblichen psychosozialen Belastungen und gesundheitlichen Konsequenzen verbunden sein [8]. Dazu zählen körperliche und seelische Verletzungen, Rückzug und Isolation, Depression, Angststörungen, soziale Beeinträchtigungen oder Substanzgebrauch [9-12]. Ergebnisse von Quer- und Längsschnittstudien zeigen, dass in Kind- heit und Jugend erfahrene Gewalt mit einem erhöhten Risiko verbunden ist, im späteren Lebensalter erneut Opfer von Gewalt zu werden, aber auch selbst Gewalt auszuüben [13-15].

Damit das Ausmaß von Gewalt eingeschätzt werden kann und Strategien zur Abmilderung der Auswirkungen entwickelt werden können, sind somit klare Definitionen von Gewalt, ein konzeptioneller Rahmen für das Verständnis der zahlreichen Erscheinungsformen und Umfelder von Gewalt sowie verlässliche Daten unabdingbar. Um die unterschiedlichen Erscheinungsformen von Gewalt angemessen abbilden und quantifizieren zu können, bedarf es zudem jeweils spezifischer Erhebungs- und Messinstrumente [2].

Die vorliegende Auswertung schließt sich an die Publikation „Körperliche und psychische Gewalterfahrungen in der deutschen Erwachsenenbevölkerung - Ergebnisse der Studie zur Gesundheit Erwachsener in Deutschland (DEGS1)“ an [13], zu der es kritische Resonanz gab [6]. Diese bezog sich insbesondere auf folgende Punkte und damit auch auf die Limitationen der in der DEGS1 enthaltenen Fragestellungen:

- Es wurde kein gendersensibles Instrument bzw. keine solche Methode verwendet, um die Erfahrung und den Umgang mit Gewalt adäquat zu erfassen. 


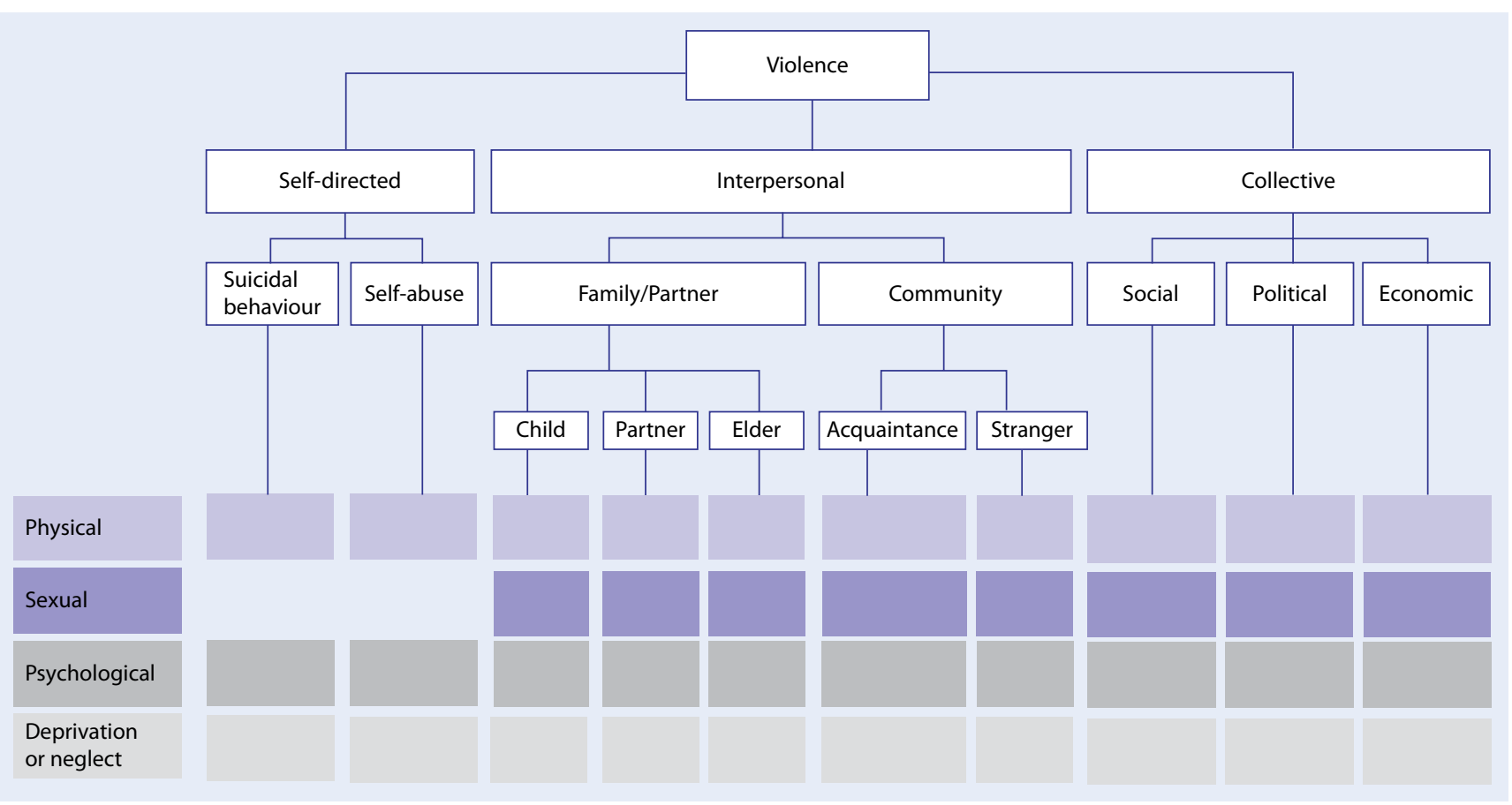

Abb. 1 A WHO-Typologie der Gewalt. Quelle:WHO-Weltbericht Gewalt und Gesundheit 2003 [2]

- Der Schweregrad der körperlichen und psychischen Gewalt wurde nicht erfasst.

- Sexuelle Gewalt wurde in die Untersuchung nicht einbezogen.

- Es wurde nicht zwischen (einmalig) im Affekt ausgeübter Gewalt und regelmäßig ausgeübter systematischer Gewalt differenziert.

- Die Folgen von Gewaltausübung wurden nicht systematisch erfasst.

Unter Berücksichtigung der genannten Limitationen sollen in der vorliegenden Publikation die Ergebnisse weitergehender Datenanalysen vorgestellt werden, wobei insbesondere folgenden, noch nicht bearbeiteten Fragestellungen nachgegangen wird:

1. Wie hoch ist der Anteil von Gewaltopfern in der Bevölkerung, denen in den letzten 12 Monaten mehrfach Gewalt widerfuhr oder die in der Folge einer Gewalterfahrung stark beeinträchtigt waren?

2. Wie hoch ist der Anteil von Personen in der Bevölkerung, die angeben, in den letzten 12 Monaten mehrfach Gewalt ausgeübt zu haben?

3. Wer waren die Konfliktpartner von mehrfachen Gewaltopfern bzw. tätern?
4. Welche Konstellationen von Gewalterfahrungen gibt es, welche soziodemografischen Merkmale haben diese Gruppen und wie ist deren gesundheitsbezogene Lebensqualität?

5. Welchen Zusammenhang gibt es zwischen ausgewählten Einflussfaktoren und dem Risiko, Gewaltopfer zu werden oder Gewalt auszuüben?

Diese Fragestellungen sollen einen vertieften und differenzierten Blick in die erhobenen Daten ermöglichen. Dabei ist einschränkend zu berücksichtigen, dass ausschließlich Fragen zu zwischenmenschlichen Gewalterfahrungen in Form von körperlicher oder psychischer Gewalt in der DEGS1 enthalten waren (- Abb. 1). Aussagen zu sexueller Gewalt, zur Schwere und Dynamik des Gewaltgeschehens, zum Kontext sowie zu Folgen von Gewalt und Gewalterfahrungen sind anhand der erhobenen Daten nicht möglich.

Ein differenziertes Gesamtbild der Gewaltausübung bzw. Gewalterfahrung bei Erwachsenen kann somit nicht erstellt werden. Trotz dieser grundsätzlichen Limitationen erlauben die Daten (Dunkelfeld) eine Einschätzung der körperlichen und psychischen Gewalterfahrung in der erwachsenen Bevölkerung im Alter zwischen 18 und 64 Jahren, die nicht allein auf angezeigten Straftaten beruht, wie sie in der polizeilichen Kriminalstatistik (Hellfeld) ausgewiesen werden.

Zudem können die Daten mit sozialen und gesundheitlichen Parametern in Beziehung gesetzt werden. Für die vorliegende Arbeit wurden, neben Alter und Geschlecht, der Sozialstatus, die Angaben zur gesundheitsbezogenen Lebensqualität und zur sozialen Unterstützung ausgewählt.

Die gesundheitsbezogene Lebensqualität, die von einem ganzheitlichen Verständnis von Gesundheit ausgeht, spiegelt den Blick der Betroffenen auf ihre eigene Gesundheit [16]. Das Konstrukt gesundheitsbezogene Lebensqualität enthält körperliche, emotionale, mentale, soziale und verhaltensbezogene Elemente des eigenen Wohlbefindens und erlaubt eine umfassende allgemeine Einschätzung der Gesundheit. Mithilfe dieses Instruments soll der Gesundheitszustand von Gewaltbetroffenen und nicht von Gewalt Betroffenen verglichen werden.

Soziale Unterstützung zählt zu den psychosozialen Ressourcen, die wesentlichen Einfluss auf die Gesundheit haben. Sie kann direkt auf das psychische Wohlbefinden wirken, Stress lindern und 
Bundesgesundheitsbl 2016 ·59:4-16 DOI 10.1007/s00103-015-2267-6

(c) Springer-Verlag Berlin Heidelberg 2015

\section{Lange $\cdot$ A. Starker $\cdot$ E. von der Lippe $\cdot$ H. Hölling}

\section{Psychische und körperliche Gewalterfahrungen in den vergangenen 12 Monaten in der Allgemein- bevölkerung. Ergebnisse der Studie zur Gesundheit Erwachsener in Deutschland (DEGS1)}

\section{Zusammenfassung}

Gewalterfahrungen können erhebliche psychosoziale und gesundheitliche Auswirkungen haben. In der bevölkerungsrepräsentativen Studie zur Gesundheit Erwachsener in Deutschland (DEGS1) wurde ein Instrument zur Erfassung körperlicher und psychischer Gewalterfahrung (sowohl aus der Opfer- und als auch aus der Täterperspektive) implementiert. Dieses Instrument wurde in der Altersgruppe 1864 Jahre ( $n=5939)$ eingesetzt. Ziel der vorliegenden Publikation ist es, den Anteil von Personen, denen innerhalb der letzten 12 Monate mehrfach Gewalt widerfuhr oder die infolge der Gewalterfahrung stark beeinträchtigt waren, zu berichten, sowie den Anteil der Personen, die mehrfach Gewalt ausgeübt haben. Des Weiteren werden die Konfliktpartner von Gewaltopfern bzw. Tätern beschrieben und besondere Konstellationen von Gewalterfahrungen in Bezug auf die gesundheitsbezogene Lebensqualität dargestellt. Abschließend wird der Zusammenhang zwischen ausgewählten Einflussfaktoren und dem Risiko, Gewaltopfer zu werden oder Gewalt auszuüben, untersucht. 2,7\% der Frauen und 4,3\% der Männer berichten von mehrfachen Erfahrungen körperlicher Gewalt in den letzten 12 Monaten oder erlebten sich durch diese Erfahrung stark beeinträchtigt; von psychischer Gewalt berichteten $18,9 \%$ der Frauen und $15,4 \%$ der Männer. Frauen sind häufiger als Männer sowohl Opfer als auch Täterin von Gewalt in der Familie. Männer berichten dagegen häufiger von Opfer- und Tätererfahrungen außerhalb des engeren familiären Umfelds. Unabhängig davon, ob sie Opfer oder
Täter körperlicher oder psychischer Gewalt waren, ist das psychische Wohlbefinden von Personen mit Gewalterfahrungen signifikant schlechter als das von Personen ohne Gewalterfahrung. Erfahrungen von Gewalt in der Kindheit und Jugend erhöhen das Risiko, später Gewaltopfer oder Gewalttäter zu werden. Die vorliegenden Ergebnisse beschreiben psychische und körperliche Gewalterfahrungen als einen Teil von Gewalthandlungen in der Allgemeinbevölkerung näher und geben so Hinweise auf mögliche Präventionspotenziale.

Schlüsselwörter

Körperliche Gewalt · Psychische Gewalt . Konfliktpartner - Gesundheitsbezogene Lebensqualität · Gesundheitssurvey

\section{Prevalence of experience of physical and psychological violence in the general population in the past 12 months. Results of the German Health Interview and Examination Survey for Adults (DEGS1)}

\section{Abstract}

Experiences of violence may have considerable psychosocial and health implications. A violence screening tool was implemented in the German Health Interview and Examination Survey for Adults (DEGS1) to depict the perpetrators' and victims' point of view. The study participants were between 18 and 64 years old $(n=5939)$. The aim of this article is to assess the percentage of people who experienced physical and psychological violence in the last 12 months or who suffered negative effects on their quality of life as a consequence or who were perpetrators of multiple acts of violence. The characteristics of victims, offenders, and their conflict partners are described. Furthermore, specific constellations of violence experience with regard to health-related quality of life are described. Finally, the association between being a victim of violence and different factors is estimated.

In total, $2.7 \%$ of women and $4.3 \%$ of men reported multiple experiences of physical violence in the last 12 months or having their lives negatively impacted as a consequence of violence. Experience of psychological violence was reported by $18.9 \%$ of women and $15.4 \%$ of men. Women are more likely than men to be both perpetrator and victim within the family. Men are more likely than women to be both the perpetrator and victim outside of the family environment. Regardless of whether they are the victim or perpetrator of violence, the psychological well-being is significantly worse than those of people who did not experience violence. Experience of violence in childhood and adolescence increases the risk of becoming victim or perpetrator of violence later on in life. The findings presented here describe the psychological and physical experience of violence as one part of violence committed in the whole population. Some prevention advice is also presented.

\section{Keywords}

Physical violence · Psychological violence . Partners of conflicts - Health related quality of life $\cdot$ Health survey die Auswirkung ungünstiger Lebensbedingungen mildern [17-20].

Die Ergebnisse der vorliegenden Untersuchung geben Informationen zum besseren Verständnis der Zusammenhänge von Gewalterfahrungen und Gesundheit. Sie lassen Zielgruppen für Präventionsbemühungen oder Unterstützungsbedarf erkennbar werden.

\section{Daten und Methoden}

DEGS ist Bestandteil des Gesundheitsmonitorings des Robert Koch-Instituts (RKI). Konzept und Design von DEGS sind an anderer Stelle ausführlich beschrieben [21, 22].

Die Analysen wurden mit einem Gewichtungsfaktor durchgeführt, der Abweichungen der Stichprobe von der Bevölkerungsstruktur (Stand 31. 12. 2010) hinsichtlich Alter, Geschlecht, Region und Staatsangehörigkeit sowie Gemeindetyp und Bildung korrigiert [23]. Um sowohl die Gewichtung als auch die Korrelation der Teilnehmenden innerhalb einer Gemeinde zu berücksichtigen, wurden die Konfidenzintervalle und pWerte mit den SPSS-20-Verfahren für komplexe Stichproben bestimmt. Nicht überlappende $95 \%$-Konfidenzintervalle 
Übersicht 1: Erfassung der psychischen und körperlichen Gewalterfahrungen in den vergangenen 12 Monaten in DEGS1

Die psychischen und körperlichen Gewalterfahrungen werden mithilfe eines Screeners erfasst, der für DEGS1 entwickelt wurde [13].

Die Fragen lauten:

\section{Körperliche Gewalterfahrungen als Opfer}

„Haben Sie in den letzten 12 Monaten erlebt, dass jemand Sie körperlich angegriffen hat? (zum Beispiel geschlagen, geohrfeigt, an den Haaren gezogen, getreten, mit einer Waffe oder einem Gegenstand bedroht)?"

Antwortkategorien: „Ja ${ }^{\prime \prime}$, Nein"

Psychische Gewalterfahrungen als Opfer

„Sind Sie in den letzten 12 Monaten durch irgendeinen Menschen abgewertet worden (z. B. bezüglich Ihres Aussehens, Ihrer Art sich zu kleiden, Ihrer Denk-, Handlungs- oder Arbeitsweise oder möglicher Behinderungen)? Oder wurden Sie beleidigt, beschimpft, bedroht, schikaniert oder unter Druck gesetzt?"

Antwortkategorien: „Ja ${ }^{\prime \prime}$, Nein"

Konfliktpartner

„Wenn ja, geschah dies durch:

Ihren Partner/Ihre Partnerin; eine Person aus der Familie, sonstige Verwandte; Kolleg(innen)en/

Vorgesetzte am Arbeitsplatz; eine sonstige, Ihnen bekannte Person; eine sonstige, Ihnen (zuvor) unbekannte Person?".

Ankreuzliste und Angabe der Häufigkeit(,"Wie oft war das?")

Belastungserleben

„Wie stark fühlten Sie sich dadurch in Ihrem Befinden beeinträchtigt?!

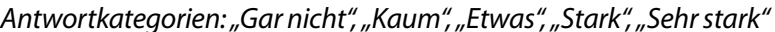

Häufigkeit von Gewalterfahrung als Erwachsener

„Haben Sie eine oder mehrere der oben beschriebenen Handlungen vorher in Ihrem Erwachsenenleben (seit dem 16. Lebensjahr) erlebt?"

Antwortkategorien: „Ja, körperliche Angriffe , ,JJa, psychisch beeinträchtigende Handlungen

oder Drohungen", "Nein, nichts davon,

Körperliche Gewalterfahrungen als Täter

„Haben Sie selbst in den letzten 12 Monaten jemanden körperlich angegriffen (zum Beispiel geschlagen, geohrfeigt, an den Haaren gezogen, getreten, mit einer Waffe oder einem Gegenstand bedroht)?"

Antwortkategorien: "Ja", "Nein"

Psychische Gewalterfahrungen als Täter

„Haben Sie in den letzten 12 Monaten eine Person abgewertet, z. B. bezüglich Ihres Aussehens, Ihrer Art sich zu kleiden, Ihrer Denk-, Handlungs- oder Arbeitsweise oder einer Behinderung? Oder haben Sie eine Person beleidigt, beschimpft, bedroht, schikaniert oder unter Druck gesetzt?"

Antwortkategorien: ${ }_{\text {,Ja }}{ }_{,{ }_{\text {,IN Nein }}}$

\section{Unrechtserleben}

„Haben Sie deswegen schon einmal ein schlechtes Gewissen gehabt oder sich schuldig gefühlt?"

Antwortkategorien: "Ja", ,Nein"

\section{Häufigkeit von Gewalterfahrung als Kind oder Jugendlicher}

„Haben Sie eine oder mehrere der folgenden Handlungen in Ihrer Kindheit und Jugend vor dem 16. Lebensjahr erfahren?

Körperliche Angriffe durch Eltern/Erziehungsberechtigte;

Körperliche Angriffe durch Gleichaltrige oder ältere Jugendliche;

Psychische Aggressionen oder Drohungen durch Eltern/Erziehungsberechtigte;

Psychische Aggressionen oder Drohungen durch Gleichaltrige oder ältere Jugendliche."

Antwortkategorien: ,"Häufig", ,Gelegentlich", ,Selten, , Nie"

werden als signifikante Unterschiede auf $\operatorname{dem} p<0,05$-Niveau gewertet.

\section{Instrumente}

Aktive und passive Erfahrungen körperlicher und psychischer Gewalt wurden im Altersbereich von 18 bis 64 Jahren bei insgesamt 5939 Teilnehmern, davon 3149 Frauen und 2790 Männer (un- gewichtete Angaben) erhoben. Eine Beschreibung der Stichprobe findet sich bei Kamtsiuris et al. [23]. Die eingesetzten Instrumente werden im Beitrag von Schlack et al. beschrieben [13]. Alle Fragen zur aktuellen Betroffenheit von Gewalt beziehen sich auf die letzten 12 Monate. Es wurde nach körperlichen und psychischen Gewalterfahrungen gefragt, wobei jeweils zunächst Opfer- und dann Tätererfahrungen erfasst wurden. Des Weiteren wurden Fragen zu Konfliktpartnern bei Gewalterfahrung, der Häufigkeit von Gewalterfahrung und zu Belastungserleben durch Viktimisierung bzw. Unrechtserleben nach Gewaltausübung erhoben. Abschließend wurde die Häufigkeit möglicher Gewalterfahrungen in der Kindheit und Jugend vor dem 16. Lebensjahr erfasst. Dazu wurden die Häufigkeit der körperlichen Angriffe und die Häufigkeit psychischer Aggressionen oder Drohungen durch Eltern/Erziehungsberechtigte bzw. Gleichaltrige/ ältere Jugendliche erfragt. Die Antwortkategorien waren „Häufig“, „Gelegentlich“, „Selten “ oder „Nie“ (• Übersicht 1).

Die ausgewählten, möglichen Einflussfaktoren auf Gewalterfahrungen wurden wie folgt erhoben:

Der Sozialstatus wurde anhand eines Indexes bestimmt, in den Angaben zu schulischer und beruflicher Ausbildung, beruflicher Stellung sowie Haushaltsnettoeinkommen (bedarfsgewichtet) eingehen und der eine Einteilung in niedrige, mittlere und hohe Statusgruppe ermöglicht [24]. Aufgrund einer Anpassung der Codierung der in der DEGS1 eingesetzten Bildungsskala Comparative Analysis of Social Mobility in Industrial Nations (CASMIN) an die Methoden des GESIS - Leibniz-Institut für Sozialwissenschaften [25] hat sich in geringem Maße die Variable des Sozialstatus geändert. Aus diesem Grund weichen die vorliegenden Ergebnisse von den bereits publizierten Daten [13] ab.

Zur Messung der gesundheitsbezogenen Lebensqualität wurde das Instrument SF 36-V2 eingesetzt. Zur Beschreibung der körperlichen und psychischen Lebensqualität wurden die Summenskalen Physical-componentScore (PCS) und Mental-componentScore (MCS) verwendet [16]. Verglichen werden dabei die Mittelwerte der Skalen der Gruppen mit Gewalterfahrungen mit denjenigen der jeweiligen Vergleichsgruppen.

Soziale Unterstützung wurde mit der Oslo-3-items-social-support-Skala gemessen $[26,27]$ und 3 Ausprägungen berechnet: Geringe, mittlere und starke Unterstützung. 


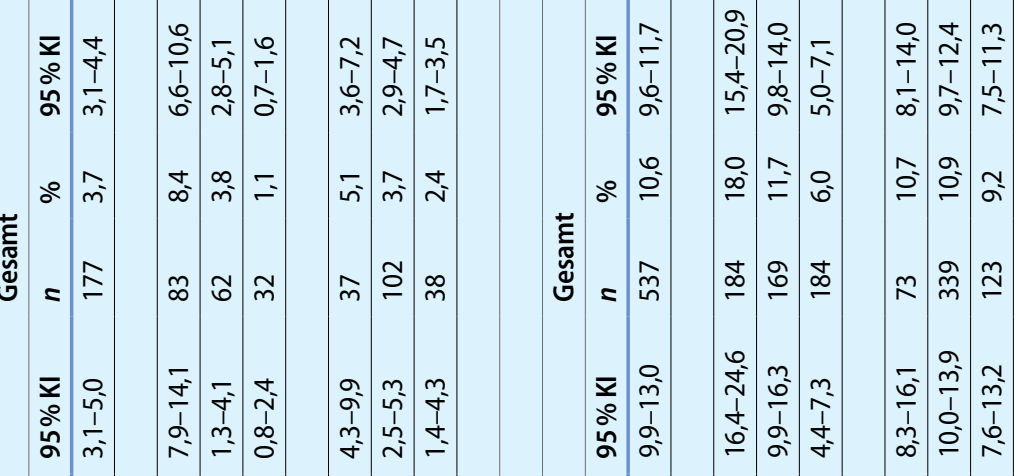

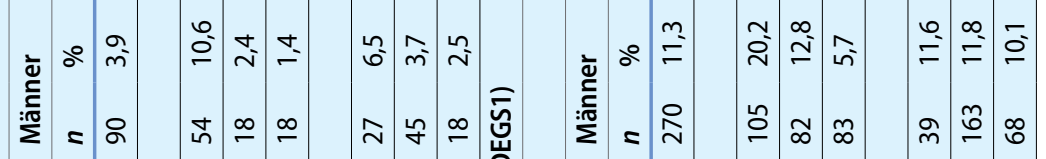

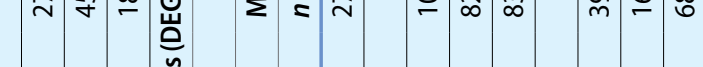

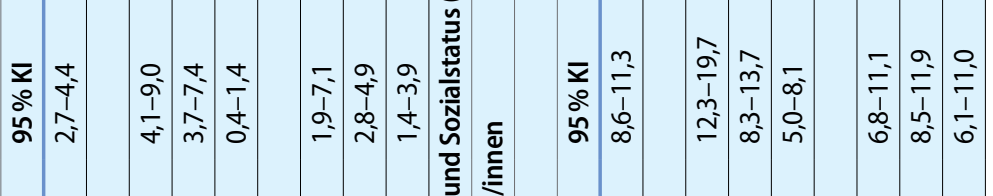

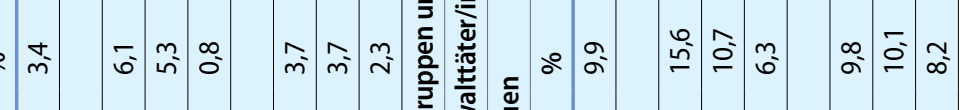

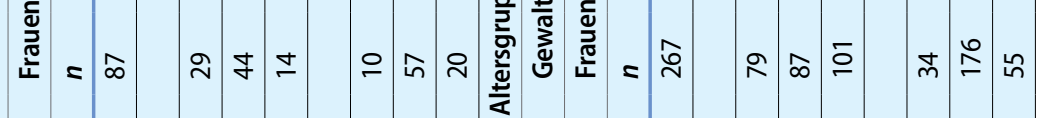

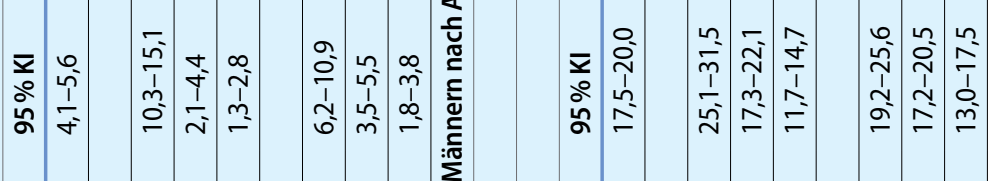

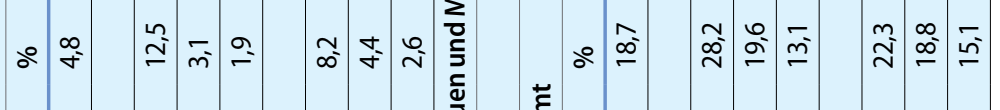

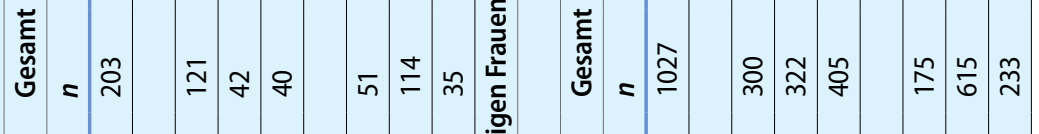

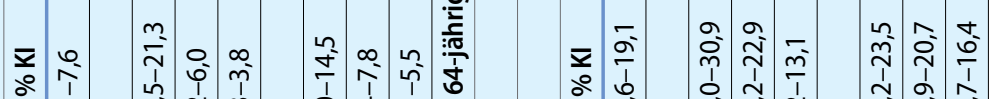

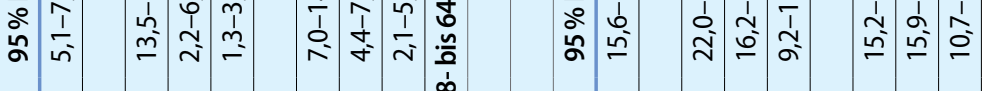

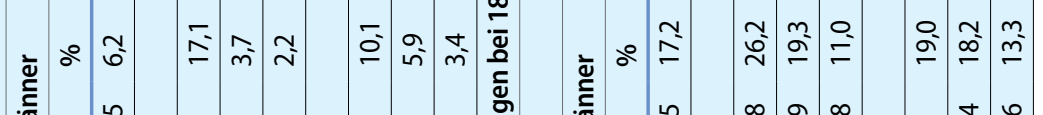

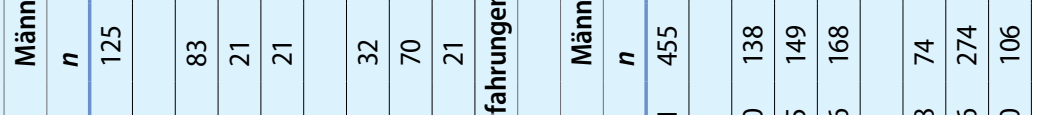

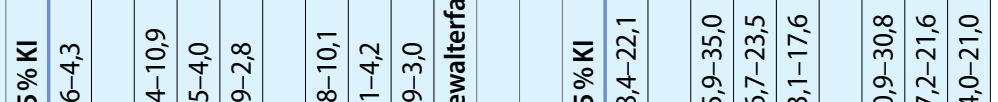

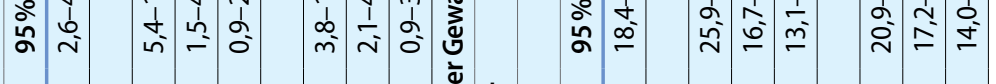

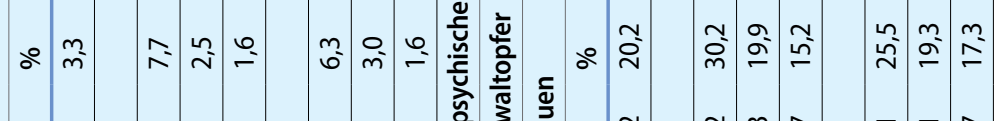

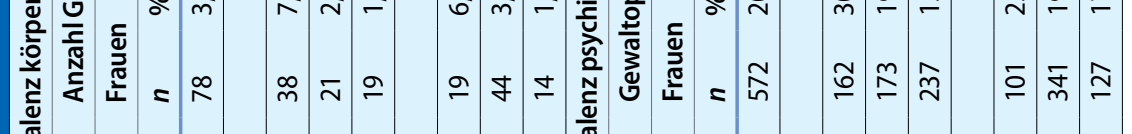




\section{Statistische Berechnungen}

Als erster Schritt erfolgt eine Grundauszählung der Daten zu körperlichen und zu psychischen Gewalterfahrungen nach Opfer- bzw. Täterstatus sowie nach Geschlecht, Alter und Sozialstatus (Reanalyse der Ergebnisse von Schlack et al. [13]).

In einem zweiten Schritt werden die Häufigkeit der körperlichen bzw. psychischen Gewalterfahrung bzw. Gewaltausübung und, im Fall der Gewalterfahrung, die Angaben zum Belastungserleben berücksichtigt. Auf Opferseite werden dazu nur die Personen betrachtet, die mehr als einmal von Gewalterfahrung (körperlich und psychisch) berichten oder durch diese stark oder sehr stark beeinträchtigt waren. Auf Täterseite werden nur diejenigen berücksichtigt, die mehr als einmal Gewalt ausgeübt haben (körperlich und psychisch). Diese Differenzierung erlaubt es, die Personen abzugrenzen, die einmalig, möglicherweise zufällig, aus Selbstschutz oder einmaligem Affekthandeln in eine gewalttätige Konflikthandlung geraten sind.

In einem dritten Schritt wird untersucht, welche Konfliktpartner von Opfern bzw. Tätern angegeben werden. Dabei wird die im Schritt 2 genannte Gruppierung der Opfer bzw. Täter nach Häufigkeit und Belastungserleben beibehalten.

In einem vierten Schritt wird eine weitere Differenzierung vorgenommen, um die Gruppe der Opfer, der Täter und der Täter/Opfer, also diejenigen, die sowohl Opfer als auch Täter bei Gewalthandlungen waren, näher zu beschreiben. Dabei wird unabhängig von der Häufigkeit und dem Belastungserleben folgende Einteilung vorgenommen:

- Opfer körperlicher und psychischer Gewalt,

- Täter körperlicher und psychischer Gewalt,

- Opfer und Täter ausschließlich körperlicher Gewalt,

- Opfer und Täter ausschließlich psychischer Gewalt.

Schwerpunkt der Auswertung ist dabei der Zusammenhang zwischen Gewalterfahrung und gesundheitsbezogener Lebensqualität.
Abschließend wird durch binär logistische Regressionsanalyse der (unabhängige) Zusammenhang zwischen möglichen Einflussfaktoren und der Tatsache, Gewaltopfer zu werden oder Gewalt auszuüben, geprüft. Es werden dafür „odds ratios“ ausgewiesen, die als Chancenverhältnisse im Vergleich zu einer Referenzkategorie zu interpretieren sind. Als mögliche Einflussfaktoren werden Alter, sozioökonomischer Status, soziale Unterstützung sowie Gewalterfahrungen in der Kindheit und Jugend geprüft, wobei die Kategorien „Häufig“ und "Gelegentlich" zusammengefasst wurden und der Gruppe „Selten“ oder „Nie“ gegenübergestellt werden.

\section{Ergebnisse}

\section{Körperliche Gewalterfahrung nach Opfer- bzw. Täterstatus}

In den letzten 12 Monaten mindestens einmal Opfer körperlicher Gewalt geworden zu sein, geben $3,3 \%$ der Frauen und 6,2 \% der Männer an. Der Unterschied zwischen den Geschlechtern ist signifikant (• Tab. 1). Mit 17,1\% sind junge Männer (18-29 Jahre alt) die Gruppe mit der höchsten Opferprävalenz. Bei Frauen wie Männern sinkt die Prävalenz mit dem Alter. Frauen und Männer aus der niedrigen sozialen Statusgruppe berichten häufiger als diejenigen aus der hohen sozialen Statusgruppe, Opfer körperlicher Gewalt geworden zu sein.

Selbst mindestens einmal in den letzten 12 Monaten körperliche Gewalt ausgeübt zu haben, geben 3,4\% der Frauen und 3,9\% der Männer an. Ebenso wie die Opferprävalenz sinkt die Täterprävalenz bei Frauen und Männern mit dem Alter. Unterschiede nach Sozialstatus zeigen sich nur bei Männern: Männer der niedrigen sozialen Statusgruppe berichten häufiger als diejenigen aus der hohen sozialen Statusgruppe, Täter körperlicher Gewalt gewesen zu sein.

\section{Psychische Gewalterfahrung nach Opfer- bzw. Täterstatus}

Mit 20,2\% der Frauen und 17,2\% der Männer wurde fast ein Fünftel der Bevölkerung im Alter zwischen 18 und
64 Jahren in den letzten 12 Monaten mindestens einmal Opfer psychischer Gewalt. Ähnlich wie bei den körperlichen Gewalterfahrungen zeigt sich eine größere Häufigkeit in den jüngeren Altersgruppen (• Tab. 1).

9,9\% der Frauen und 11,3\% der Männer haben innerhalb der letzten 12 Monate mindestens einmal eine andere Person psychisch abgewertet oder beleidigt. Die Prävalenz der psychischen Gewaltausübung sinkt bei Frauen und Männern mit dem Alter. Zwischen den sozialen Statusgruppen gibt es keine Unterschiede in der Prävalenz psychischer Gewaltausübung.

\section{Gewalterfahrung und Gewaltausübung unter Berücksichtigung der Häufigkeit und des Belastungserlebens}

Vier Fünftel (82,7\%; 95 \% KI 71,7-82,7; $n=64)$ der Frauen mit körperlichen Gewalterfahrungen wurden mehrfach innerhalb der letzten 12 Monate Opfer oder waren durch diese Erfahrung stark beeinträchtigt; bei Männern trifft dies auf gut zwei Drittel (68,5\%; $95 \%$ KI 58,1-77,3; $n=82)$ der Opfer körperlicher Gewalt zu.

Eine wiederholte Täterschaft unter Einsatz körperliche Gewalt berichten jeweils knapp die Hälfte der Frauen $(47,4 \%$; $95 \%$ KI 35,9-59,2; $n=43$ ) und Männer $(49,6 \%$; $95 \%$ KI $36,2-63,1 ; n=44)$.

Mehrfach innerhalb der letzten 12 Monate psychisch abgewertet oder beleidigt oder durch diese Erfahrung stark beeinträchtigt waren $93,9 \%$ (95\% KI 91,1$95,8 ; n=539)$ der Frauen und $89,5 \%$ (95\% KI 85,6-92,4; $n=403$ ) der Männer mit Erfahrungen psychischer Gewalt.

Von den Personen, die angaben, in den letzten 12 Monaten psychische Gewalt ausgeübt zu haben, haben dies $73,5 \%$ (95\% KI 66,2-79,7; $n=188$ ) der Frauen und 78,8\% $(95 \% \mathrm{KI} 72,3-84,1 ; n=211)$ der Männer mehrfach getan. Insgesamt tritt wiederholte Gewalterfahrung und Täterschaft bei psychischer Gewalt häufiger auf als bei körperlicher Gewalt.

Im Vergleich zu der Grundauswertung (• Tab. 1), bei der die Häufigkeit der Gewalt und das Belastungserleben nicht mit einbezogen wurden, unterscheiden sich bei mehrfacher Gewalterfahrung bzw. Gewaltausübung die Muster zwischen 


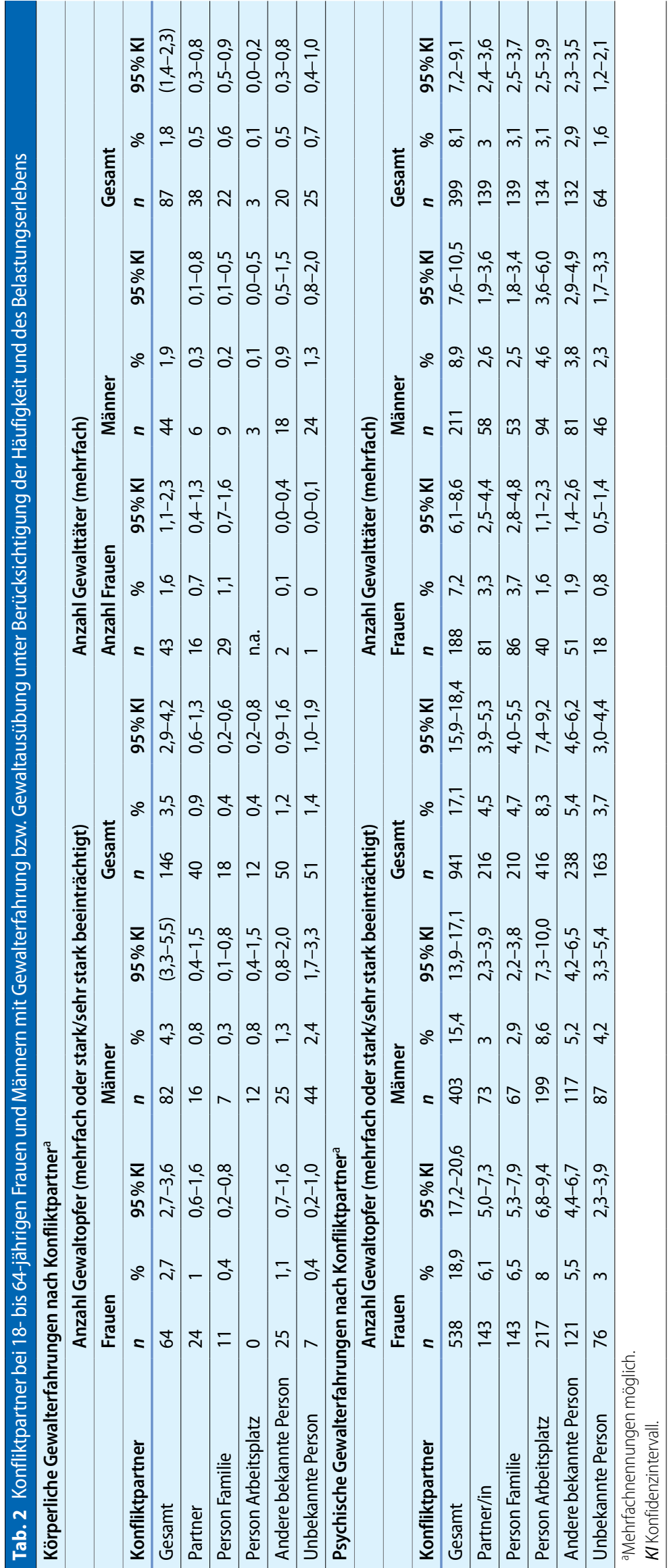

Geschlecht, Alter und Sozialstatus nicht wesentlich; die Prävalenzen von Gewalterfahrung und Gewaltausübung sind aber insgesamt geringer: $2,7 \%$ der Frauen (95\% KI 2,1-3,6; $n=64)$ und $4,3 \%$ der Männer (95\% KI 3,3-5,5; $n=82$ ) berichten von mehrfachen Erfahrungen körperlicher Gewalt in den letzten 12 Monaten oder starker Beeinträchtigung infolge der Gewalterfahrung. Eine mehrfache Täterschaft geben 1,6\% der Frauen (95\% KI 1,1-2,3; $n=43$ ) und $1,9 \%$ der Männer (95\% KI 1,3-2,8; $n=44$ ) an. Mehrfach in den letzten 12 Monaten psychische Gewalt erfahren haben 18,9\% der Frauen (95\% KI 17,3-20,7; $n=539$ ) und 15,4\% der Männer (95\% KI 13,8-17,2; $n=403)$. Selbst mehrfach andere Personen abgewertet oder beleidigt haben $7,2 \%$ der Frauen $(95 \% \mathrm{KI} 6,1-8,6 ; n=188)$ und $8,9 \%$ der Männer (95\% KI 7,6-10,5; $n=211$ ).

\section{Konfliktpartner mehrfacher körperlicher und psychischer Gewalt}

Im Folgenden wird betrachtet, welche Konfliktpartner von den Personen angegeben wurden, die mehrfach körperliche oder psychische Gewalt erfahren oder ausgeübt hatten (s. vorheriger Abschnitt). Eine Übersicht über die bevölkerungsbezogenen Prävalenzen mehrfacher körperlicher und psychischer Gewalterfahrungen nach Konfliktpartnern findet sich in - Tab. 2.

Aufgrund der insgesamt geringen Fallzahl von Personen mit mehrfacher körperlicher Gewalterfahrung (Opferund Tätererfahrungen), der Möglichkeit von Mehrfachnennungen und aufgrund fehlender Erfassung des Kontexts sind differenzierte Aussagen zu den Konfliktpartnern nur eingeschränkt möglich. Es lassen sich aber Muster feststellen, die sich zwischen Frauen und Männern unterscheiden und einen Hinweis auf unterschiedliche Gewaltexpositionen geben.

Frauen erlitten mehrfach körperliche Gewalt etwa zur Hälfte durch Familienmitglieder (Partner oder eine andere Person aus der Familie), zur anderen Hälfte durch eine andere bekannte oder unbekannte Person. Bei männlichen Opfern waren zu etwa zwei Dritteln Täter oder Täterin eine bekannte oder un- 


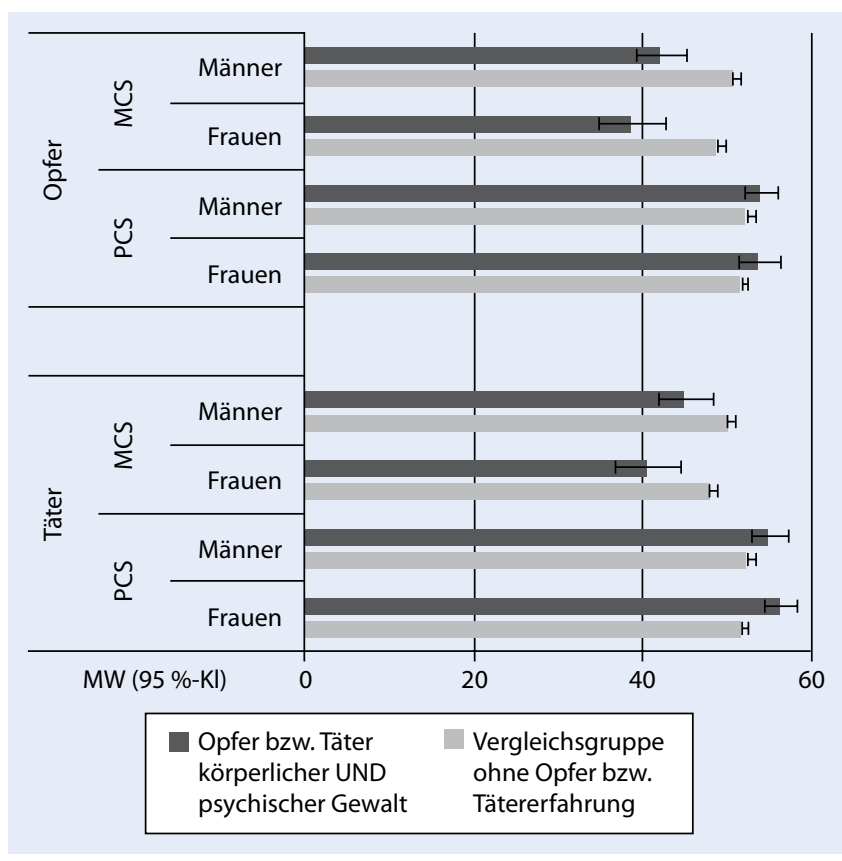

Abb. $2 \Delta$ Mittelwerte des Mental-component-Score (MCS) und des Physical-component-Score (PCS) von Personen, die Opfer $(n=124)$ bzw. Täter $(n=80)$ sowohl von körperlicher als auch psychischer Gewalt waren, im Vergleich zu Personen ohne Opfer- bzw. Tätererfahrung (unabhängig von der Häufigkeit der Gewalterfahrung und dem Belastungserleben)

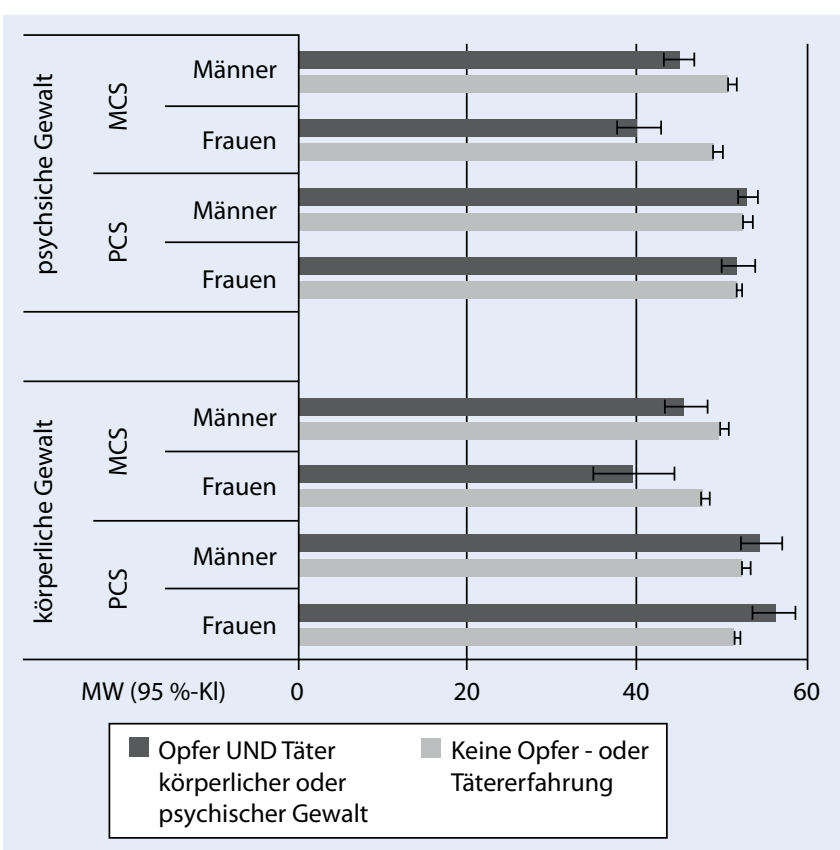

Abb. $3 \Delta$ Mittelwerte des Mental-component-Score (MCS) und des Physical-component-Score (PCS) von Personen, die sowohl Opfer als auch Täter von körperlicher $(n=75)$ oder psychischer Gewalt $(n=334)$ waren, im Vergleich zu Personen, die weder Opfer noch Täter waren (unabhängig von der Häufigkeit der Gewalterfahrung und dem Belastungserleben) bekannte Person, zu gut einem Fünftel kamen Täter oder Täterin aus der Familie, in jedem 9. Fall stammte der Täter oder die Täterin aus dem Arbeitsumfeld (Kollegen oder Vorgesetzte).

Die Täterinnen übten in 9 von 10 Fällen körperliche Gewalt an Familienmitgliedern aus (Partner oder andere Angehörige); bei Männern dagegen betraf die von ihnen ausgeübte körperliche Gewalt überwiegend andere bekannte und unbekannte Personen, in jedem 4. Fall Personen aus der Familie (Partner oder andere Person aus der Familie) und in jedem 20. Fall eine Person aus dem Arbeitsumfeld (Kollegen oder Vorgesetzte). Frauen sind somit häufiger als Männer sowohl Opfer als auch Täterin von Gewalt im engeren sozialen Umfeld (Partner und Familie). Männer dagegen berichten häufiger als Frauen Opfer- und Tätererfahrungen außerhalb des engeren familiären Umfelds.

Mehrfache psychische Viktimisierung erfuhren Frauen in 4 von 10 Fällen durch Familienangehörige (Partner oder andere Familienmitglieder), in jeweils etwa 3 von 10 Fällen durch andere bekannte oder unbekannte Personen und durch Arbeits- kollegen. In drei Viertel der Fälle, in denen Männer abgewertet oder beleidigt wurden, geschah dies durch eine andere bekannte oder unbekannte Person oder durch eine Person aus dem Arbeitsumfeld. Etwa ein Viertel der Beleidigungen oder Abwertungen erfolgte durch eine Person aus der Familie (Partner oder anderes Familienmitglied).

Psychische Gewalt übten Frauen in 6 von 10 Fällen gegenüber Familienangehörigen (Partner oder anderes Familienmitglied) aus, zu knapp einem Viertel gegenüber einer anderen bekannten oder unbekannten Person und in jedem 7. Fall gegenüber Arbeitskollegen. Bei Männern waren die Opfer psychischer Gewalt in 3 von 10 Fällen Familienangehörige, in 4 von 10 Fällen eine andere bekannte oder unbekannte Person und in knapp jedem 3. Fall eine Person aus dem Arbeitsumfeld.

\section{Konstellationen von Gewalterfahrungen}

Interpersonelle Gewalt wird häufig nicht nur als körperliche oder psychische Gewalt ausgeübt, sondern tritt in Konflikt- situationen vielfach zusammen bzw. hintereinander auf (,anschreien und schlagen") oder aber auch reziprok (das Opfer wehrt sich und wird zum Täter oder umgekehrt; vgl. auch Meuser 2006 zum „reziproken Typus der Männergewalt" $[28,29])$. Deswegen werden im Folgenden weitere Differenzierungen nach Konstellationen von Gewalt vorgenommen.

\section{Opfer von körperlicher und psychischer Gewalt}

Innerhalb der letzten 12 Monate sowohl Opfer körperlicher als auch psychischer Gewalt geworden zu sein, gaben 56 Frauen $(2,4 \%$; $95 \%$ KI 1,8-3,2) und 68 Männer $(3,3 \% ; 95 \%$ KI $2,4-4,4)$ an. Die Gewaltopfer sind jünger als nicht von Gewalt Betroffene. Frauen und Männer mit Gewalterfahrungen gehören signifikant häufiger niedrigen Sozialstatusgruppen an als Personen ohne Gewalterfahrungen.

\section{Täter von körperlicher und psychischer Gewalt}

Körperliche und psychische Gewalt übten 39 Frauen $(1,5 \%$; $95 \%$ KI $1,1-2,1)$ und 41 Männer (1,7\%; $95 \%$ KI 1,2-2,5) aus. 
Tab. 3 Zusammenhänge zwischen verschiedenen Einflussfaktoren und mehrfacher Opfererfahrung körperlicher oder psychischer Gewalt in den letzten 12 Monaten $(n=997)$. Ergebnisse binär logistischer Regressionen

\begin{tabular}{|c|c|c|c|c|c|c|}
\hline & \multicolumn{3}{|c|}{ Männer } & \multicolumn{3}{|c|}{ Frauen } \\
\hline & OR & $95 \% \mathrm{KI}$ & $p$ & OR & $95 \% \mathrm{KI}$ & $p$ \\
\hline \multicolumn{7}{|l|}{ Alter } \\
\hline 18-29 Jahre & 3,1 & $2,18-4,40$ & 0,000 & 2,34 & $1,76-3,11$ & 0,000 \\
\hline 30-44 Jahre & 1,94 & $1,38-2,72$ & 0,000 & 1,35 & $1,02-1,78$ & 0,037 \\
\hline 45-64 Jahre & \multicolumn{6}{|c|}{ Diente als Referenz } \\
\hline \multicolumn{7}{|c|}{ Sozioökonomischer Status } \\
\hline Niedrig & \multicolumn{6}{|c|}{ Diente als Referenz } \\
\hline Mittel & 1,02 & $0,72-1,44$ & 0,923 & 0,91 & $0,63-1,30$ & 0,599 \\
\hline Hoch & 0,86 & $0,57-1,30$ & 0,475 & 0,89 & $0,57-1,36$ & 0,578 \\
\hline \multicolumn{7}{|l|}{ Soziale Unterstützung } \\
\hline Gering & \multicolumn{6}{|c|}{ Diente als Referenz } \\
\hline Mittel & 0,69 & $0,47-0,99$ & 0,047 & 0,63 & $0,43-0,93$ & 0,022 \\
\hline Stark & 0,56 & $0,36-0,87$ & 0,010 & 0,49 & $0,31-0,77$ & 0,002 \\
\hline \multicolumn{7}{|c|}{ Häufigkeit der Gewalterfahrungen in Kindheit und Jugend } \\
\hline \multicolumn{7}{|c|}{ Körperliche Angriffe/Eltern } \\
\hline Nie/Selten & \multicolumn{6}{|c|}{ Diente als Referenz } \\
\hline Gelegentlich/Häufig & 1,04 & $0,68-1,61$ & 0,850 & 1,46 & $0,95-2,27$ & 0,086 \\
\hline \multicolumn{7}{|c|}{ Körperliche Angriffe/Peers } \\
\hline Nie/Selten & \multicolumn{6}{|c|}{ Diente als Referenz } \\
\hline Gelegentlich/Häufig & 2,1 & $1,48-2,97$ & 0,000 & 1,4 & $0,82-2,38$ & 0,212 \\
\hline \multicolumn{7}{|c|}{ Psychische Aggressionen/Eltern } \\
\hline Nie/Selten & \multicolumn{6}{|c|}{ Diente als Referenz } \\
\hline Gelegentlich/Häufig & 2,28 & $1,46-3,55$ & 0,000 & 2,52 & $1,65-3,84$ & 0,000 \\
\hline \multicolumn{7}{|c|}{ Psychische Aggressionen/Peers } \\
\hline Nie/Selten & \multicolumn{6}{|c|}{ Diente als Referenz } \\
\hline Gelegentlich/Häufig & 1,69 & $1,19-2,41$ & 0,003 & 2,48 & $1,69-3,65$ & 0,000 \\
\hline
\end{tabular}

Die Täter sind deutlich jünger als die Vergleichsgruppe; die Täter gehören signifikant häufiger niedrigen Sozialstatusgruppen an als die Vergleichsgruppe ohne Täterschaft.

\section{Opfer und Täter ausschließlich körperlicher Gewalt}

Gleichermaßen Opfer und Täter körperlicher Gewalt waren 23 Frauen $(1,1 \%$; $95 \%$ KI 0,7-1,6) und 52 Männer (2,5\%; $95 \%$ KI 1,8-3,5). Die Verteilung nach Alters- und Sozialstatusgruppen entspricht den anderen, oben beschriebenen Gruppen. Der bevölkerungsbezogene Anteil von Männern ist in dieser Gruppe doppelt so hoch wie der von Frauen.

\section{Opfer und Täter ausschließlich psychischer Gewalt}

Opfer und Täter psychischer Gewalt gewesen zu sein, berichteten 165 Frauen
$(6,3 \%$; $95 \%$ KI 5,4-7,5) und 169 Männer (6,7\%; $95 \%$ KI 5,6-8,0). Diese Gruppe ist ebenfalls jünger als die Vergleichsgruppe; Unterschiede nach sozialen Statusgruppen sind aber nicht signifikant.

Schwerpunkt dieser Auswertungen ist der Zusammenhang von Gewalterfahrungen und gesundheitsbezogener Lebensqualität. In allen Konstellationen von Personen mit Gewalterfahrungen (Opfer- wie Tätererfahrungen) ist das psychische Wohlbefinden, gemessen durch die Summenskala MCS, signifikant niedriger als das der Vergleichsgruppe ohne Gewalterfahrungen (• Abb. 2, 3). Diese Unterschiede zeigen sich nicht bei einem Vergleich der Mittelwerte der Summenskala für das körperliche Wohlbefinden (PCS).

Es fällt aber auf, dass Frauen, die Täterinnen körperlicher und psychischer Gewalt waren und Frauen, die Opfer oder Täterin körperlicher Gewalt waren, ein besseres körperliches Wohlbefinden (PCS) berichten als die Vergleichsgruppe ohne Gewalterfahrungen. Bei einer Interpretation der Daten ist jedoch zu berücksichtigen, dass sich die Alterszusammensetzung der Personen mit Gewalterfahrungen von derjenigen der jeweiligen Vergleichsgruppen unterscheidet.

\section{Einflussfaktoren von Gewalterfahrungen}

Mithilfe binär logistischer Regressionsanalysen wurde abschließend untersucht, inwieweit mögliche Einflussfaktoren wie Alter, sozioökonomischer Status, soziale Unterstützung sowie Gewalterfahrungen in der Kindheit und Jugend mit Gewalterfahrungen in den letzten 12 Monaten in Zusammenhang stehen (- Tab. 3 und 4). Um statistische Analysen mit ausreichender Präzision durchführen zu können (Fallzahl), wurden hier mehrfache Opfer körperlicher oder psychischer Gewalt $(n=997)$ sowie mehrfache Täter körperlicher oder psychischer Gewalt $(n=440)$ jeweils zu einer Gruppe zusammengefasst. Getrennt nach Geschlecht wird dabei das Risiko (statistisch korrekt die Chance) für körperliche oder psychische Gewalt als Opfer oder Täter im Vergleich zu einer Referenzgruppe ohne entsprechende Gewalterfahrungen berechnet. Für die Auswertungen der Häufigkeit möglicher Gewalterfahrungen in der Kindheit und Jugend wurden die Kategorien „Häufig“ und „Gelegentlich“"zusammengefasst und der Gruppe „Selten“ oder „Nie“ gegenübergestellt.

Das Risiko, Opfer einer Gewalttat zu werden, ist in den jüngeren Altersgruppen (18-44 Jahre) bei Frauen wie bei Männern signifikant höher als in der Altersgruppe 45-64 Jahre. Der sozioökonomische Status steht in keinem statistisch signifikanten Zusammenhang mit der Opfererfahrung. Mittlere und hohe soziale Unterstützung reduzieren das Risiko, Gewaltopfer zu werden. Bei Männern erhöhen die Erfahrungen körperlicher Angriffe Gleichaltriger vor dem 16. Lebensjahr, ebenso wie psychische Aggressionen durch Eltern oder Gleichaltrige das Risiko, Gewaltopfer zu werden, um den 
Tab. 4 Zusammenhänge zwischen verschiedenen Einflussfaktoren und mehrfacher Tätererfahrung körperlicher oder psychischer Gewalt in den letzten 12 Monaten $(n=440)$. Ergebnisse binär logistischer Regressionen

\begin{tabular}{|c|c|c|c|c|c|c|}
\hline & \multicolumn{3}{|c|}{ Männer } & \multicolumn{3}{|c|}{ Frauen } \\
\hline & OR & $95 \mathrm{KI}$ & $p$ & OR & $95 \mathrm{KI}$ & $p$ \\
\hline \multicolumn{7}{|l|}{ Alter } \\
\hline 18-29 Jahre & 5,18 & $3,41-7,87$ & 0,000 & 3,27 & $2,10-5,08$ & 0,000 \\
\hline 30-44 Jahre & 2,19 & $1,37-3,49$ & 0,001 & 1,95 & $1,30-2,92$ & 0,001 \\
\hline 45-64 Jahre & \multicolumn{6}{|c|}{ Diente als Referenz } \\
\hline \multicolumn{7}{|c|}{ Sozioökonomischer Status } \\
\hline Niedrig & \multicolumn{6}{|c|}{ Diente als Referenz } \\
\hline Mittel & 1,25 & $0,75-2,08$ & 0,386 & 1,03 & $0,62-1,69$ & 0,919 \\
\hline Hoch & 1,21 & $0,66-2,22$ & 0,537 & 0,89 & $0,49-1,64$ & 0,714 \\
\hline \multicolumn{7}{|l|}{ Soziale Unterstützung } \\
\hline Gering & \multicolumn{6}{|c|}{ Diente als Referenz } \\
\hline Mittel & 0,63 & $0,35-1,12$ & 0,112 & 1,33 & $0,74-2,40$ & 0,340 \\
\hline Stark & 0,65 & $0,35-1,21$ & 0,169 & 1,11 & $0,60-2,08$ & 0,732 \\
\hline \multicolumn{7}{|c|}{ Häufigkeit der Gewalterfahrungen in Kindheit und Jugend } \\
\hline \multicolumn{7}{|c|}{ Körperliche Angriffe/Eltern } \\
\hline Nie/Selten & \multicolumn{6}{|c|}{ Diente als Referenz } \\
\hline Gelegentlich/Häufig & 0,99 & $0,55-1,76$ & 0,969 & 1,32 & $0,71-2,45$ & 0,378 \\
\hline \multicolumn{7}{|c|}{ Körperliche Angriffe/Peers } \\
\hline Nie/Selten & \multicolumn{6}{|c|}{ Diente als Referenz } \\
\hline Gelegentlich/Häufig & 2,21 & $1,35-3,62$ & 0,002 & 0,92 & $0,51-1,61$ & 0,781 \\
\hline \multicolumn{7}{|c|}{ Psychische Aggressionen/Eltern } \\
\hline Nie/Selten & \multicolumn{6}{|c|}{ Diente als Referenz } \\
\hline Gelegentlich/Häufig & 2,07 & $1,13-3,80$ & 0,019 & 3,03 & $1,77-5,18$ & 0,000 \\
\hline \multicolumn{7}{|c|}{ Psychische Aggressionen/Peers } \\
\hline Nie/Selten & \multicolumn{6}{|c|}{ Diente als Referenz } \\
\hline Gelegentlich/Häufig & 1,27 & $0,72-2,24$ & 0,401 & 2,32 & $1,33-4,06$ & 0,003 \\
\hline
\end{tabular}

Faktor 2. Bei Frauen trifft dies nur auf erfahrene psychische Aggressionen in der Kindheit und Jugend durch Eltern oder Gleichaltrige zu.

Männer im Alter von 18 bis 29 Jahren haben ein um den Faktor 5 erhöhtes Risiko, Gewalt auszuüben, im Vergleich zu Männern im Alter von 45 bis 64 Jahren. Auch bei Frauen ist das Risiko der Gewaltausübung in den jüngeren Altersgruppen signifikant höher als in der höchsten Altersgruppe. Weder der sozioökonomische Status noch die soziale Unterstützung zeigen einen eindeutigen statistischen Zusammenhang zu einer Täterschaft. Bei Männern ist das Risiko, Gewalt auszuüben, um das 2-Fache erhöht, wenn sie körperliche Angriffe von Gleichaltrigen oder psychische Aggressionen durch Eltern in der Jugend erfahren hatten. Bei Frauen besteht dieser Zusammenhang im Hinblick auf
Abgrenzung zur amtlichen Statistik, die allein auf angezeigten Straftaten beruht, ermöglichen die Daten von DEGS1 jedoch eine Beschreibung der Mehrfachopfer bzw. Mehrfachtäter in Beziehung zu sozialen und gesundheitlichen Parametern.

Auf Basis der vorliegenden Daten wurde zunächst die Häufigkeit körperlicher und psychischer Gewaltausübung beschrieben, um einen Überblick zu geben und den Bezugsrahmen für die weiteren Auswertungen zu verdeutlichen. Doppelt so viel Männer wie Frauen (6,2 versus $3,3 \%$ ) berichteten, in den letzten 12 Monaten mindestens einmal Opfer körperlicher Gewalt geworden zu sein; nach eigenen Angaben selbst körperliche Gewalt ausgeübt haben 3,4\% der Frauen und 3,9\% der Männer. Vergleichbare 12-Monats-Prävalenzen wurden auch in Studien aus Neuseeland und den USA gefunden [30-32]. Nach Ergebnissen des European Survey of Crime and Safety (EU ICS) 2005 [33] betrug die 12-Monats-Prävalenz von Gewaltopfererfahrungen (Tätlichkeiten und Bedrohungen) in Deutschland knapp $3 \%$. Da Personen ab 16 Jahren in die Erhebung einbezogen waren und die Prävalenz von Gewalterfahrungen mit dem Alter sinkt, liegen die Ergebnisse in der Größenordnung der DEGS1Ergebnisse.

Mindestens einmal von psychischer Gewalt betroffen waren 20,2\% Frauen und 17,2\% Männer. Selbst eine andere Person abgewertet oder beleidigt haben 9,9\% der Frauen und 11,3\% der Männer. Vergleichszahlen zu Verbreitung psychischer Gewalt auf Bevölkerungsebene liegen nicht vor [13], der Fokus der Untersuchungen liegt meist auf der Erfassung von Partnergewalt. Eine aktuelle populationsbezogene Studie zu Partnergewalt aus Belgien fand eine 12-MonatsPrävalenz von $14 \%$ bei psychologischer Gewalt. Zwischen Frauen und Männern bestand dabei kein Unterschied [34].

Mehr Frauen als Männer wurden mehrfach Opfer körperlicher Gewalt oder waren durch diese Erfahrung beeinträchtigt. Mit diesem Ergebnis korrespondiert, dass Frauen auch häufiger als Männer durch Familienmitglieder Gewalt erfahren. Dies kann als Hinweis auf die Chronifizierung von Gewalt in engen 
sozialen Beziehungen gedeutet werden [35]. Jede 2. Person, die körperliche Gewalt ausübte, tat dies mehr als einmal. Jeweils etwa 9 von 10 Opfern psychischer Gewalt waren wiederholt betroffen; drei Viertel der Personen, die psychische Gewalt ausübten, taten dies mehrfach.

Eine Betrachtung der Konfliktpartner von Personen mit mehrfacher Gewalterfahrung zeigt unterschiedliche Muster bei Männern und Frauen. Frauen sind häufiger als Männer sowohl Opfer als auch Täter von Gewalt in der Familie (Partner oder andere Familienangehörige). Männer berichten dagegen häufiger von Opfer- und Tätererfahrungen außerhalb des engeren familiären Umfelds. Arbeitskolleginnen und Kollegen sind nicht selten Ziel von Beleidigungen oder Abwertungen, sowohl aus der Täter- als auch aus der Opferperspektive. Entsprechende Erfahrungen aus der Arbeitswelt berichten Männer häufiger als Frauen. In der Europäischen Erhebung über die Arbeitsbedingungen [European Working Conditions Survey (EWCS)] wurden im Jahr 2010 Angaben zu Erfahrungen mit Gewalt am Arbeitsplatz erhoben, wobei allerdings nur Opfererfahrungen erfasst wurden. Danach zeigt sich für Deutschland, dass nahezu gleich viele Frauen wie Männer von diesen Erfahrungen berichten [36].

Unabhängig davon, ob sie Opfer oder Täter körperlicher oder psychischer Gewalt waren, und unabhängig von verschiedenen Konstellationen von Gewalterfahrungen ist das psychische Wohlbefinden von Personen mit Gewalterfahrung signifikant schlechter als das von Personen ohne Gewalterfahrung. Bei dem körperlichen Wohlbefinden lassen sich anhand der DEGS1-Daten keine entsprechenden Unterschiede zeigen. Dass Gewalterfahrungen mit psychischen Gesundheitsproblemen einhergehen, zeigen auch andere Studien [34, 37, 38].

Erfahrungen von Gewalt in der Kindheit und Jugend - durch psychische Aggressionen von Eltern und Erziehungsberechtigten bzw. durch körperliche Angriffe oder psychische Aggressionen von Gleichaltrigen oder älteren Jugendlichen erhöhen das Risiko, später Gewaltopfer oder Gewalttäter zu werden. Körperliche Angriffe von Eltern und Er- ziehungsberechtigten zeigen dagegen keinen signifikanten Zusammenhang zu späterer Gewalterfahrung. Hohe soziale Unterstützung ist mit einem niedrigeren Risiko, Gewaltopfer zu werden, assoziiert. Auch andere Studien weisen darauf hin, dass sowohl familiäre als auch Gewalterfahrungen durch Peers entscheidenden Einfluss auf Gewalteinstellungen und tatsächliches Gewalthandeln von Jugendlichen haben [39, 40]. Vernachlässigung oder Misshandlung von Kindern und Jugendlichen kann einen Kreislauf von Gewalt („cycle of violence“) zur Folge haben. Als Protektivfaktoren werden soziale Sicherheit und soziale Unterstützung angesehen [41]. Für Präventionsmaßnahmen wird ein lebensphasenbezogener Ansatz vorgeschlagen, der das gesamte Umfeld der Familie einbezieht [39].

Aus den Ergebnissen können auch Hinweise auf mögliche Präventionspotenziale abgeleitet werden. Dazu gehören beispielsweise gezielte Maßnahmen zur Gewaltprävention im Jugendalter, im jungen Erwachsenenalter oder im familiären Umfeld. Dabei sollte v. a. berücksichtigt werden, dass Gewaltausübung häufig mit erhöhtem Alkoholkonsum (Risikokonsum oder Rauschtrinken) verbunden ist. Laut polizeilicher Kriminalstatistik 2014 wurden 29,4\% der aufgeklärten Gewaltdelikte von Tatverdächtigen unter Alkoholeinfluss begangen [42]. Ergebnisse von Van Dijk et al. [33] belegen die Korrelation von Gewalttaten und Bierkonsum. Eine Sekundäranalyse der Schweizer ESPAD-Studie (Europäische Schülerstudie zu Alkohol und anderen Drogen) bei Jugendlichen im Alter von 13 bis 17 Jahren zeigt, dass Gewalt bei Alkoholkonsumierenden, insbesondere Risikokonsumierenden, häufiger vorkommt. Der alkoholattributive Anteil an Gewalt wird für Jungen zwischen $25 \%$ und $50 \%$ geschätzt, für Mädchen zwischen $30 \%$ und über $70 \%$ [43]. Nach dieser Studie üben Jungen zwar mehr Gewaltakte aus als Mädchen, wenn es aber zu Gewalt kommt, ist bei Mädchen häufiger Alkohol im Spiel. Es wird daher die Verschränkung von strukturellen, individuellen und schadensreduzierenden Maßnahmen zur Alkohol- und Gewaltprävention sowie eine Verbesserung der Früherkennung vorgeschlagen. Die DEGS1-Daten belegen, dass in der Altersgruppe der 18- bis 29-Jährigen nicht nur die Prävalenz von Gewalterfahrungen am höchsten ist, sondern auch die Prävalenz von Rauschtrinken und Alkoholrisikokonsum [44] und somit auch für diese Altersgruppe verschränkte Präventionsansätze sinnvoll erscheinen.

Eine Übersicht über den Stand und die weltweite Umsetzung von Präventionsmaßnahmen gegen Gewalt sowie Empfehlungen zur Prävention gibt die WHO in einem aktuellen Report [45].

Personen, die durch eine Gewalttat eine gesundheitliche Beeinträchtigung erfahren haben, können nach dem Opferentschädigungsgesetz (OEG) auf Antrag Heilbehandlungs-, Renten- und Fürsorgeleistungen erhalten [46]. Zum Leistungskatalog gehören u. a. Heil- und Krankenbehandlungen (einschließlich Psychotherapie). Die DEGS1-Daten belegen das signifikant schlechtere psychische Wohlbefinden von Personen mit Gewalterfahrungen - daher sollten bei der Behandlung und Unterstützung von Personen mit Gewalterfahrungen jeweils auch psychotherapeutische Interventionen geprüft werden. Statistiken des Weißen Rings zur Opferentschädigung belegen allerdings, dass nur rund $10 \%$ der Anspruchsberechtigten einen Antrag nach OEG stellen [47]. Diese geringe Inanspruchnahme beruht $u$. a. auf dem geringen Bekanntheitsgrad des OEG. Somit erhalten nur wenige Betroffene eine spürbare Hilfe bei der Bewältigung körperlicher, psychischer oder wirtschaftlicher Tatfolgen.

\section{Limitationen}

In DEGS1 wurden ausschließlich Fragen zu zwischenmenschlichen Gewalterfahrungen in Form von körperlicher oder psychischer Gewalt erhoben. Aussagen zur Verbreitung von Gewalt gegen die eigene Person und Aussagen zur Verbreitung kollektiver Gewalt können somit nicht gemacht werden. Des Weiteren wurden in DEGS1 aus bereits beschriebenen Gründen nicht alle Formen der Gewaltausübung erfasst [13]. Damit ist nur ein Teil des Gewaltgeschehens beschreibbar und die Vergleichbar- 
keit zu anderen Studien eingeschränkt. Limitationen liegen weiterhin in der Tatsache, dass die Fallzahlen zu gering sind, um weitere Differenzierungen bei der Beschreibung der psychischen und körperlichen Gewalterfahrung vornehmen $\mathrm{zu}$ können. Die Altersbegrenzung auf die 18- bis 64-Jährigen ermöglicht zudem keine Aussagen darüber, in welchem Umfang und in welcher Weise ältere Menschen von Gewalt betroffen sind. Weitere Einschränkungen betreffen die Studienpopulation: An der Studie haben Personen teilgenommen, die grundsätzlich bereit sind, Informationen über ihre Gesundheit und ihre Lebensumstände preiszugeben. Personen, die schwer durch Gewalterfahrungen traumatisiert sind oder kriminelle Handlungen begehen, sind durch bevölkerungsbezogene Surveys schwer zu erreichen („selection bias"). Es ist zudem nicht auszuschließen, dass Aspekte sozialer Erwünschtheit zum Tragen kamen und die Befragten nicht oder nicht vollständig über ihre Gewalterfahrungen berichtet haben. Auch Scham über das Erlebte bzw. die eigene Gewaltausübung kann eine Rolle gespielt haben („reporting bias“). Zudem ist auch ein Genderbias in Bezug auf die Selbstangaben von Gewalthandlungen zu vermuten: Handlungen, die innerhalb der eigenen Geschlechtsrolle als normal akzeptiert und nicht als Gewalt kategorisiert werden, werden vermutlich auch nicht berichtet. So werden teilweise körperliche Auseinandersetzungen innerhalb des eigenen Geschlechts oder gegenüber Frauen als konstituierender Teil des männlichen Rollenbilds interpretiert. Männliches Gewalthandeln verstößt zwar gegen die Rechtsordnung, wird aber nicht selten von Geschlechtsgenossen akzeptiert. Weibliches Gewalthandeln dagegen steht im Widerspruch zu kulturellen Erwartungen und zur Rechtsordnung. Diese „Geschlechtslogik“ von Gewalt kann dazu beitragen, dass das Potenzial der Gewalt überwiegend von Männern realisiert wird [28, 29].

Finanzierung der DEGS1 Studie. Die Studie wurde mit Mitteln des Robert Koch-Instituts und des Bundesministeriums für Gesundheit finanziert.

\section{Korrespondenzadresse}

\section{Dr. C. Lange}

Abteilung für Epidemiologie nichtübertragbarer Krankheiten und Gesundheitsmonitoring

Robert Koch-Institut

General-Pape-Str. 62-66, 12101 Berlin

LangeC@rki.de

\section{Einhaltung ethischer Richtlinien}

Interessenkonflikt. C. Lange, A. Starker, E. von der Lippe, H. Hölling geben an, dass keine Interessenkonflikte bestehen.

Das DEGS Studienprotokoll wurde von der EthikKommission der Charité Universitätsmedizin Berlin gebilligt und vom Bundesbeauftragten für den Datenschutz und die Informationsfreiheit für unbedenklich erachtet. Alle Teilnehmenden gaben schriftlich nach umfassender Aufklärung ihre Einwilligung in die Befragung und die Untersuchungen.

\section{Literatur}

1. Imbusch P (2002) Der Gewaltbergriff. In: Heitmeyer W, Hagan J (Hrsg) Internationales Handbuch der Gewaltforschung. Westdeutscher, Wiesbaden, S 26-57

2. Weltgesundheitsorganisation (WHO) (2003) Weltbericht Gewalt und Gesundheit. Zusammenfassung. WHO Regionalbüro für Europa, Kopenhangen

3. World Health Organization (WHO) (1996) Global Consultation on Violence and Health. Violence: a public health priority. WHO, Geneva

4. Bundesministerium für Familie, Senioren, Frauen und Jugend (BMFSFJ) (2004) Gewalt gegen Männer in Deutschland. Personale Gewaltwiderfahrnisse von Männern in Deutschland. Pilotstudie. Im Auftrag des Bundesministerium für Familie, Senioren, Frauen und Jugend. BMFSFJ, Berlin

5. Schröttle M (2010) Kritische Anmerkungen zur These der Gendersymmetrie bei Gewalt in Paarbeziehungen. Gend Z Geschlecht Kult Ges Heft 1:133-151

6. Brzank P, Blattner B, Hahn D (2013) Valide Aussagen zu Gewalt im Geschlechterverhältnis erfordern gendersensible Erfassung. Bundesgesundheitsblatt Gesundheitsforschung Gesundheitsschutz 56(9):1335-1336

7. Brzank P (2012) Wege aus der Partnergewalt Frauen auf der Suche nach Hilfe. VS Verlag, Wiesbaden

8. Robert Koch Institut (RKI) (2014) Gesundheitliche Lage der Männer in Deutschland. Beiträge zur Gesundheitsberichterstattung des Bundes, Kapitel 3.6 Gewalt. RKI, Berlin

9. European Commission (2011) The State of Men's Health in Europe - Extended Report. European Union, Brüssel

10. Scarpa A (2001) Community Violence Exposure in a Young Adult Sample. J Inter-personal Violence 16(1):36-53

11. Sundaram V, Helweg-Larsen $K$, Laursen B et al (2004) Physical violence, self rated health, and morbidity: is gender significant for victimisation? J Community Health 58(1):65-70
12. Olofsson N, Lindqvist K, Shaw BA et al (2012) Longterm health consequences of violence exposure in adolescence: a 26-year prospective study. BMC Public Health 12:1

13. Schlack R, Rüdel J, Karger A et al (2013) Körperliche und psychische Gewalterfahrungen in der deutschen Erwachsenenbevölkerung: Ergebnisse der Studie zur Gesundheit von Erwachsenen in Deutschland (DEGS1). Bundesgesundheitsblatt Gesundheitsforschung Gesundheitsschutz 56(56):755-764

14. Fang X, Corso PS (2007) Child Maltreatment, Youth Violence, and Intimate Partner Violence. Developmental Relationships. Am J Prev Med 33(4):281-290

15. Manly JT, Kim JE, Rogosch FA et al (2001) Dimensions of child maltreatment and children's adjustment: contributions of developmental timing and subtype. Dev Psychopathol 13(4):759782

16. Ellert U, Kurth BM (2013) Gesundheitsbezogene Lebensqualität bei Erwachsenen in Deutschland. Ergebnisse der Studie zur Gesundheit Erwachsener in Deutschland (DEGS1). Bundesgesundheitsblatt Gesundheitsforschung Gesundheitsschutz 56(5-6):643-649

17. Robert Koch Institut (RKI) (2014) Daten und

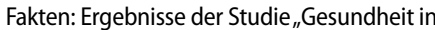
Deutschland aktuell 2012". Beiträge zur Gesundheitsberichterstattung des Bundes. RKI, Berlin

18. Berkman LF, Glass T, Brissette I et al (2000) From social integration to health: Durkheim in the new millennium. Soc Sci Med 51(6):843-857

19. Uchino BN (2006) Social support and health: a review of physiological processes potentially underlying links to disease outcomes. J Behav Med 29(4):377-387

20. Uchino BN, Cacioppo JT, Kiecolt-Glaser JK (1996) The relationship between social support and physiological processes: a review with emphasis on underlying mechanisms and implications for health. Psychol Bull 119(3):488-531

21. Scheidt-Nave C, Kamtsiuris $P$, Göwald $A$ et al (2012) German health interview and examination survey for adults (DEGS) - design, objectives and implementation of the first data collection wave. BMC Public Health 12(1):730

22. Gößwald A, Lange $M$, Kamtsiuris $P$ et al (2012) DEGS: Studie zur gesundheit Erwachsener in Deutschland: Bundesweite Quer- und Längsschnittstudie im Rahmen des Gesundheitsmonitorings des Robert Koch-Instituts. Bundesgesundheitsblatt Gesundheitsforschung Gesundheitsschutz 55(6-7):775-780

23. Kamtsiuris $P$, Lange $M$, Hoffmann R et al (2013) Die erste Welle der Studie zur Gesundheit Erwachsener in Deutschland (DEGS1): Stichprobendesign, Response, Gewichtung und Repräsentativität. Bundesgesundheitsblatt Gesundheitsforschung Gesundheitsschutz 56(5-6):620630

24. Lampert T, Kroll L, Müters S et al (2013) Messung des sozioökonomischen Status in der Studie zur Gesundheit Erwachsener in Deutschland (DEGS 1). Bundesgesundheitsblatt Gesundheitsforschung Gesundheitsschutz 56(5-6):631-636

25. Lechert $Y$, Schroedter J, Lüttinger P (2006) Die Umsetzung der Bildungsklassifikation CASMIN für die Volkszählung 1970, die Mikrozensus-Zusatzerhebung 1971 und die Mikrozensen 1976-2004. ZUMA-Methodenbericht 2006/12. Mannheim

26. Dalgard OS, Bjork S, Tambs K (1995) Social support, negative life events and mental health. Br J Psychiatry 166:29-34 


\section{Leitthema}

27. Meltzer H (2003) Development of a common instrumnet for mental health. In: Nosikov A, Gudex C (Hrsg) EUROHIS: developing common instruments for health surveys. IOS Press, Amsterdam

28. Meuser M (2002) „Doing Masculinity“ - Zur Geschlechtslogik männlichen Gewalthandelns. In: Dackweiler R-M, Schäfer R (Hrsg) Gewaltverhältnisse Feministische Perspektiven auf Geschlecht und Gewalt. Campus Verlag, Frankfurt a.M., S 5378

29. Meuser M (2006) Männliche Sozialisation und Gewalt. In: Berliner Forum Gewaltprävention (Hrsg) Dokumentation des 6 Berliner Präventionstages am 24 November 2005; BFG Nr 24, Berlin, S 15-22

30. Gelles R (2002) Gewalt in der Familie. In: Heitmeyer W, Hagan J (Hrsg) Internationales Handbuch der Gewaltforschung. Westdeutscher Verlag, Wiesbaden, S 1043-1077

31. Fanslow J, Robinson E, Crengle S et al (2010) Juxtaposing beliefs and reality: prevalence rates of intimate partner violence and attitudes to violence and gender roles reported by New Zealand women. Violence Against Women 16(7):812-831

32. Catalano S (2013) Intimate Partner Violence: attributes of victimization, 1993-2011. Bureau of Justice Statistics, Washington

33. Van Dijk JJM, Manchin R, van Kesteren JN et al (2005) The burden of crime in the EU, A comparative analysis of the European survey of crime and safety (EU ICS) 2005. http://www.unicri. it/services/library_documentation/publications/ icvs/publications/EUICS_-_The_Burden_of_ Crime_in_the_EU.pdf. Zugegriffen: 13. August 2015

34. Hellemans S, Buysse A, De Smet O et al (2014) Intimate partner violence in Belgium: prevalence, individual health outcomes, and relational correlates. Psychol Belg 54(1):79-96

35. Blätter B (2015) Gewalt - ein Public Health Thema. Präv Z Gesundh 38(2):35-37

36. Eurofond (2012) Fifth European Working Conditions Survey. Publications Office of the European Union, Luxembourg

37. Hellemans S, Loeys T, Dewitte M et al (2015) Prevalence of intimate partner violence victimization and victims' relational and sexual well-being. J Famil Violence 30:685-698

38. Kostev K, Rex J, Waehlert L et al (2014) Risk of psychiatric and neurological diseases in patients with workplace mobbing experience in Germany: a retrospective database analysis. Ger Med Sci 12:Doc10. doi:10.3205/000195

39. Pfeiffer C, Wetzels P, Enzmann D (1999) Innerfamiliäre Gewalt gegen Kinder und Jugendliche und ihre Auswirkungen. Kriminologisches Forschungsinstitut Niedersachsen e. V., Hannover

40. Wetzels P (1997) Gewalterfahrungen in der Kindheit. Sexueller Missbrauch, körperliche Misshandlung und deren langfristige Konsequenzen. Nomos Verlagsgesellschaft, Baden-Baden

41. World Health Organization (WHO) (2007) The cycles of violence. The relationship between childhood maltreatment and the risk of later becoming a victim or perpetrator of violence. WHO Regional Office for Europe, Copenhagen

42. Bundeskriminalamt (BKA) (2015) Polizeiliche Kriminalstatistik 2014. BKA, Wiesbaden

43. Kuntsche E, Gmel G, Annaheim B (2006) Alkohol und Gewalt im Jugendalter. Gewaltformen aus Täter- und Opferperspektive, Konsummuster und Trinkmotive - Eine Sekundäranalyse der ESPADSchülerbefragung. Schweizerische Fachstelle für Alkohol- und andere Drogenprobleme, Lausanne
44. Hapke U, V. Der Lippe E, Gaertner B (2013) Riskanter Alkoholkonsum und Rauschtrinken unter Berücksichtigung von Verletzungen und der Inanspruchnahme alkoholspezifischer medizinischer Beratung: Ergebnisse der Studie zur Gesundheit Erwachsener in Deutschland (DEGS1). Bundesgesundheitsblatt Gesundheitsforschung Gesundheitsschutz 56(5-6):809-813

45. World Health Organization (WHO) (2014) Global status report on violence prevention 2014. WHO, Luxembourg

46. Bundesministerium für Arbeit und Soziales (BMAS) (2010) Opferentschädigungsrecht. www. bmas.de/DE/Themen/Soziale-Sicherung/SozialeEntschaedigung/Opferentschaedigungsrecht/oeg html. Zugegriffen: 14. August 2015

47. Weisser Ring e. V. (2015) Statistiken zur staatlichen Opferentschädigung. www.weisser-ring. de/internet/medien/statistiken-zur-staatlichenopferentschaedigung/index.html. Zugegriffen: 14 August 2015 